\title{
The association between serum adenosine deaminase levels and Graves' disease
}

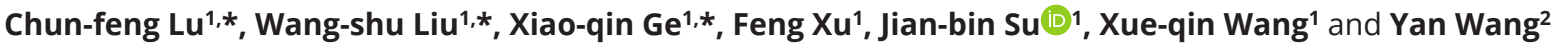 \\ 1'Department of Endocrinology, Affiliated Hospital 2 of Nantong University and First People's Hospital of Nantong City, Nantong, China \\ 2Department of Geriatrics, Affiliated Hospital 2 of Nantong University and First People's Hospital of Nantong City, Nantong, China
}

Correspondence should be addressed to F Xu or J Su or X Wang: xufeng5205529@163.com or sujbzjx@163.com or wangxueqin108@163.com

*(C Lu, W Liu and X Ge contributed equally to this work)

\begin{abstract}
Background: Adenosine deaminase (ADA) is essential for the differentiation and maturation of lymphocytes, while lymphocytes infiltration in thyroid tissue is a vital pathological feature of Graves' disease (GD). The aim of the present study was to compare the concentration of ADA between healthy controls $(H C)$ and patients with GD, and evaluate the association between ADA and GD.

Methods: A total of 112 GD patients and 77 matched HC were enrolled in this study. Each participant was examined for thyroid hormones and autoantibodies, ADA concentration, and thyroid ultrasonography.

Results: Serum ADA levels in GD patients were significantly higher than that in $\mathrm{HC}$ subgroup $(P<0.001)$. In GD patients, serum ADA levels were positively associated with serum-free triiodothyronine (FT3), free thyroxine (FT4), thyroid peroxidase antibody (TPOAb), thyroid-stimulating hormone receptor antibody (TRAb) levels, and total thyroid gland volume (thyroid VolT) and negatively associated with serum thyroid-stimulating hormone receptor (TSH) levels (all $P<0.05$ ). There were no similar correlations in the HC subgroup. Multiple linear regression analysis suggested that serum TSH, FT3, and ADA levels played an important role in serum TRAb levels.

Conclusions: Our results demonstrated that serum ADA levels were closely associated with GD.
\end{abstract}

Key Words

- adenosine deaminase

- Graves' disease

- thyroid-stimulating hormone receptor antibody

\section{Introduction}

Graves' disease (GD) characterized by diffuse goiter, thyrotoxicosis, and ophthalmopathy is an organ-specific autoimmune disease, with a prevalence of about 1\% (1). GD is a multifactorial disease that genetic predisposition, environmental factors, and immune system dysfunction are all implicated in GD pathogenesis, among which immune system dysfunction plays the most important role (2). As an autoimmune disease, relapsing cases of GD are common, and remission time is uncertain, which may create difficulties in treating this disease (3). Treatments for GD include oral antithyroid drugs, radioactive iodine therapy (RAI), and surgery, but each treatment regimen has the possibility of side effects (4). From this statement, further understanding of the mechanism of immune system dysfunction in GD pathogenesis and exploring new therapeutic targets are of great importance for improving the prognosis of GD patients.

Adenosine deaminase (ADA) is a polymorphic enzyme which is expressed in all human tissues. In addition to regulating adenosine concentration by catalyzing the irreversible deamination of adenosine to inosine (5), ADA is also essential for the differentiation and maturation of $\mathrm{T}$ lymphocytes and is a general marker of cellular immunity (6). In some autoimmune diseases, such as psoriasis (7), autoimmune hepatitis (8), inflammatory bowel disease (9), and rheumatic disease (10),
This work is licensed under a Creative Commons Attribution-NonCommercial 4.0 International License. ded from Bioscientifica.com at 04/26/2023 07:32:02AM 
high serum concentrations of ADA were observed. Meanwhile, increased ADA activity was also observed in the peripheral blood leucocytes of patients with GD (11) and monocytes of patients with Hashimoto disease (12). Therefore, serum ADA level may have the potential to be an indicator for the evaluation and monitoring of GD. Moreover, in patients with psoriasis, treatment with propylthiouracil (PTU), a kind of oral antithyroid drugs, significantly reduced the epidermal ADA activity while improving psoriatic plaques (13). Based on this, we speculated that PTU might play a role in the treatment of hyperthyroidism partly by inhibiting the activity of ADA, so ADA might be a potential therapeutic target for GD. However, to our knowledge, there are no clinical studies that have explored the relationship between serum ADA levels and GD.

Therefore, the present study was designed to compare the concentration of ADA between HC and GD patients, and to evaluate the association between ADA and GD.

\section{Methods}

\section{Participants' selection}

This was a cross-sectional study, and a total of 112 GD patients at the inpatient department of the Second Affiliated Hospital of Nantong University between January 2021 and April 2021 were enrolled. GD was defined as: clinical symptoms of hyperthyroidism, characteristic of thyroid ultrasound images, and biochemical indicators (each criterion must be met: low thyroid-stimulating hormone - TSH, high free triiodothyronine - FT3, high free thyroxine - FT4, elevated TSH receptor antibody - TRAb) (14). The exclusion criteria were as follows: (1) complicated with other autoimmune diseases; (2) use of drugs in the past 3 months that affect immunity, i.e. steroids; (3) pregnancy, previous and current malignant tumors; and (4) abnormal liver function due to various causes. During the same period, the present study also included 77 age- and sex-matched healthy controls from the Department of Physical Examination Center. The study protocol was approved by the medical research ethics committee of the Second Affiliated Hospital of Nantong University, and completely complied with the Declaration of Helsinki. Upon enrollment, each subject provided a written informed consent.

\section{Basic data collection}

Clinical data including age, sex, smoking, medical history, and anthropometry parameters were obtained from all participants through interviews and examinations by experienced physicians. BMIwas calculated as the weight $(\mathrm{kg}) /$ height $\left(\mathrm{m}^{2}\right)$. After resting for at least $30 \mathrm{~min}$, blood pressure of each participant was measured by a standard mercury sphygmomanometer, and the average of three recordings were recorded.

\section{Laboratory examination}

After an overnight fasting, venous blood samples were drawn to measure laboratory parameters. Serum ADA concentrations were measured with an automated biochemical analyzer (Model 7600, Hitachi). Serum TSH, FT3, FT4, TRAb, thyroid peroxidase antibody (TPOAb), and thyroglobulin antibody (TgAb) levels were determined using chemiluminescence methods with an immunoassay system (DxI800, Beckman Coulter).

\section{Statistical analysis}

Clinical variables were shown for all subjects, GD subgroup, HC subgroup, and for the three subgroups of GD patients according to ADA tertiles. The mean \pm S.D, median (25 and $75 \%$ interquartile), and frequency (percentage) were adopted to describe continuous variables with normal and skewed distributions and categorical variables, respectively. We adopted Student's $t$-test to compare differences in normally distributed data, the Mann-Whitney test to compare differences in skewed distributed data, and the chi-square test to compare categorical data between GD subgroup and HC subgroup. One-way ANOVA, the Kruskal-Wallis test, and the chisquare test were used to compare differences in normally distributed data, skewed data, and categorical data among the three subgroups of GD patients based on ADA tertiles. Spearman's bivariate correlation analysis was constructed to evaluate the correlations of ADA levels with clinical parameters in GD subgroup and HC subgroup, respectively. Since the distribution of serum TRAb level was skewed, a natural logarithm transformation (ln) was applied to achieve a normal distribution. Subsequently, multiple linear regression analysis was undertaken to identify independent contributors to $\ln$ (TRAb). Furthermore, receiver operating characteristic (ROC) analysis was conducted to analyze the ability of ADA levels to indicate GD cases, and the corresponding cut-off value was provided. Data analyses were performed using SPSS statistical software 18.0 (IBM SPSS Inc.). A value of $P<0.05$ was considered to be statistically significant. 


\section{Results}

\section{Basic characteristics}

Table 1 displays the clinical characteristics of the participants. Compared with HC, GD patients had higher ADA concentrations, FT3 levels, FT4 levels, TPOAb levels, $\mathrm{TgAb}$ levels, TRAb levels, total thyroid gland volume (thyroid VolT), lower BMI, and TSH levels (all $P<0.001$ ). There were no differences in age, proportion of males, proportion of smoking, and systolic/diastolic blood pressure $(P>0.05)$ between the two subgroups.

GD patients were then divided into three subgroups according to the ADA tertiles, and clinical indicators were compared between the subgroups. As shown in Table 2, along with the ADA tertile ascending, age, FT3 levels, FT4 levels, TPOAb levels, TRAb levels, and thyroid VolT significantly increased $(P$ for trend $<0.05$ ), whereas diastolic blood pressure (DBP) and TSH levels decreased ( $P$ for trend < 0.05). However, proportion of males, proportion of smoking, proportion of relapsed case, proportion of antithyroid treatment, BMI, systolic blood pressure (SBP), and TgAb levels did not show any difference among the tertiles of ADA levels ( $P$ for trend $>0.05$ ).

\section{Relationship between ADA and clinical parameters}

Table 3 shows that in GD subgroup, serum ADA levels were positively associated with age, FT3 levels, FT4 levels, TPOAb levels, TRAb levels, and thyroid VolT $(r=0.298, r=0.378, r=0.379, r=0.209, r=0.518, r=0.421$, respectively, all $P<0.05)$ and negatively associated with DBP and TSH levels $(r=-0.248, r=-0.262$, respectively, both $P<0.05)$. However, there were no significant correlations between ADA levels and male, smoking, relapsed case, antithyroid treatment, BMI, SBP, or TgAb levels (all $P>0.05$ ). In HC subgroup, only a significant positive correlation between serum ADA levels and age $(r=0.273, P<0.05)$ was observed.

\section{Multiple linear regression analysis with In (TRAb) as the dependent variable in GD subgroup}

To explore which parameters were independent contributors to TRAb, we constructed a multiple linear regression analysis. After adjusting for age, male, smoking, relapsed case, antithyroid therapy, BMI, SBP, DBP, FT4, TPOAb, TgAb and thyroid VolT, TSH levels $(\beta=-0.470$, $t=-2.951, \quad P=0.004)$, FT3 levels $(\beta=0.064, \quad t=2.501$, $P=0.005)$, and ADA levels $(\beta=0.118, t=4.478, P<0.001)$ were independently correlated with $\ln (\mathrm{TRAb})$ (Table 4$)$.

\section{ROC analysis to explore the cut-off ADA value to diagnose GD cases}

ROC analysis was further conducted to explore the cutoff ADA value to indicate confirmed GD cases. The optimal cutoff value of ADA to indicate GD was $10 \mathrm{U} / \mathrm{L}$. The corresponding area under the curve (AUC) to indicate GD was 0.808 (95\% CI: 0.746-0.869), its Youden index

Table 1 Clinical characteristics of the study participants.

\begin{tabular}{lc}
\hline Variables & Total \\
\cline { 1 - 1 } Age (years) & 189 \\
Male, $n(\%)$ & $47.7 \pm 13.79$ \\
Smoking, $n(\%)$ & $33(17.5)$ \\
BMI (kg/m²) & $22.77 \pm 3.37$ \\
SBP $(\mathrm{mmHg})$ & $123.0(115.0-138.5)$ \\
DBP (mmHg) & $77.10 \pm 10.58$ \\
TSH (mlu/L) & $0.01(0.01-1.71)$ \\
FT3 (IU/L) & $5.85(4.91-14.74)$ \\
FT4 (IU/L) & $14.81(10.83-46.26)$ \\
TPOAb (IU/mL) & $40.50(1.60-436.98)$ \\
TgAb (IU/mL) & $1.7(0.1-20.9)$ \\
TRAb (IU/L) & $4.13(0.80-12.16)$ \\
Thyroid VolT (mL) & $24.23(16.78-39.50)$ \\
ADA (U/L) & $9(7-12)$ \\
\end{tabular}

\begin{tabular}{c} 
GD \\
\hline 112 \\
$47.65 \pm 14.37$ \\
$17(15.2)$ \\
$4(3.6)$ \\
$22.04 \pm 3.24$ \\
$123.5(116.3-141.5)$ \\
$76.20 \pm 10.49$ \\
$0.01(0.01-0.01)$ \\
$13.04(7.40-20.03)$ \\
$40.56(20.72-53.65)$ \\
$218.15(9.70-671.58)$ \\
$3.9(0.5-36.2)$ \\
$8.54(4.12-20.46)$ \\
$30.39(20.52-45.13)$ \\
$11(9-14)$
\end{tabular}

\begin{tabular}{c}
\hline HC \\
\hline 77 \\
$47.78 \pm 12.99$ \\
$16(20.8)$ \\
$3(3.9)$ \\
$23.83 \pm 3.29$ \\
$122.0(113.5-135.0)$ \\
$78.40 \pm 10.65$ \\
$1.91(1.34-2.79)$ \\
$4.88(4.48-5.31)$ \\
$10.95(9.88-11.62)$ \\
$1.3(0.6-9.2)$ \\
$0.1(0.1-3.1)$ \\
$0.80(0.58-0.80)$ \\
$16.63(20.52-20.21)$ \\
$8(7-9)$
\end{tabular}

\begin{tabular}{r}
\hline P value \\
\hline \\
0.950 \\
0.211 \\
0.599 \\
$<0.001$ \\
0.240 \\
0.160 \\
$<0.001$ \\
$<0.001$ \\
$<0.001$ \\
$<0.001$ \\
$<0.001$ \\
$<0.001$ \\
$<0.001$ \\
$<0.001$
\end{tabular}

Normally distributed values in the table are given as the mean \pm S.D., skewed distributed values are given as the median ( 25 and $75 \%$ interquartiles), and categorical variables are given as frequency (percentage). The two independent sample $t$-test, the Mann-Whitney test and the chi-square test were conducted to determine $P$ values for normally distributed continuous variables, skewed continuous variables, and categorical variables, respectively. ADA, adenosine deaminase; FT4, free thyroxine; FT3, free triiodothyronine; SBP/DBP, systolic/diastolic blood pressure; TgAb, thyroglobulin antibody; TPOAb, thyroid peroxidase antibody; TRAb, TSH receptor antibody; TSH, thyroid-stimulating hormone; thyroid VolT, total thyroid gland volume.

https://ec.bioscientifica.com https://doi.org/10.1530/EC-21-0344 (c) 2021 The authors Published by Bioscientifica Ltd

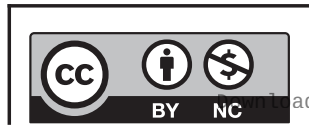

This work is licensed under a Creative Commons Attribution-NonCommercial 4.0 International License. ded from Bioscientifica.com at 04/26/2023 07:32:02AM 
Table 2 Clinical characteristics of GD patients based on ADA tertiles.

\begin{tabular}{l}
\hline Variables \\
\hline ADA (U/L) \\
$n$ \\
Age (years) \\
Male, $n(\%)$ \\
Smoking, $n(\%)$ \\
Relapsed case, $n(\%)$ \\
Antithyroid treatment \\
$\quad$ None, $n(\%)$ \\
Propylthiouracil, $n(\%)$ \\
$\quad$ Methimazole, $n(\%)$ \\
BMI (kg/m²) \\
SBP (mmHg) \\
DBP (mmHg) \\
TSH (mlu/L) \\
FT3 (IU/L) \\
FT4 (IU/L) \\
TPOAb (IU/mL) \\
TgAb (IU/mL) \\
TRAb (IU/L) \\
Thyroid VolT (mL)
\end{tabular}

\begin{tabular}{c} 
T1 \\
\hline$<10$ \\
37 \\
$44.05 \pm 15.19$ \\
$6(16.2)$ \\
$2(5.4)$ \\
$6(16.7)$ \\
$25(69.4)$ \\
$2(5.6)$ \\
$9(25.0)$ \\
$22.10 \pm 3.47$ \\
$125.0(116.0-141.0)$ \\
$79.73 \pm 10.96$ \\
$0.01(0.01-0.01)$ \\
$8.88(5.70-14.45)$ \\
$22.73(13.59-335.58)$ \\
$114.2(15.1-335.6)$ \\
$1.8(0.3-22.0)$ \\
$4.37(1.70-6.96)$ \\
$24.16(13.96-34.67)$
\end{tabular}

\begin{tabular}{c} 
T2 \\
$10-12$ \\
35 \\
$45.46 \pm 12.21$ \\
$7(20.0)$ \\
$1(2.9)$ \\
$7(20.6)$ \\
$19(55.9)$ \\
$2(5.9)$ \\
$13(38.2)$ \\
$21.99 \pm 3.09$ \\
$121.0(114.0-134.0)$ \\
$76.60 \pm 10.37$ \\
$0.01(0.01-0.01)$ \\
$14.10(8.53-19.92)$ \\
$43.18(29.95-49.83)$ \\
$258.9(5.70-645.0)$ \\
$4.9(0.5-23.9)$ \\
$7.93(4.84-15.00)$ \\
$29.0(19.89-43.19)$ \\
\hline
\end{tabular}

\begin{tabular}{|c|c|}
\hline T3 & $P$ for trend \\
\hline \multicolumn{2}{|l|}{$>12$} \\
\hline 40 & \\
\hline $52.90 \pm 14.14$ & 0.013 \\
\hline $4(10.0)$ & 0.473 \\
\hline $1(2.5)$ & 0.761 \\
\hline $7(17.5)$ & 0.905 \\
\hline $28(70.0)$ & 0.622 \\
\hline $3(7.5)$ & 0.622 \\
\hline $9(22.5)$ & 0.622 \\
\hline $22.03 \pm 3.26$ & 0.989 \\
\hline $126.5(117.0-146.0)$ & 0.448 \\
\hline $72.58 \pm 9.13$ & 0.010 \\
\hline $0.01(0.01-0.01)$ & 0.009 \\
\hline $17.10(9.51-24.67)$ & 0.002 \\
\hline $47.61(28.79-59.94)$ & 0.002 \\
\hline $350.40(58.8-905.2)$ & $<0.001$ \\
\hline $7.9(0.5-52.5)$ & 0.358 \\
\hline $19.93(9.08-26.20)$ & $<0.001$ \\
\hline $39.25(25.20-52.46)$ & 0.005 \\
\hline
\end{tabular}

ANOVA, the Mann-Whitney test, and the chi-square test were conducted to determine $P$ values for normally distributed continuous variables, skewed continuous variables, and categorical variables, respectively.

was 0.475 , its sensitivity was $67.0 \%$, and its specificity was 80.5\% (Fig. 1).

\section{Discussion}

In the present study, we compared serum ADA levels and explored the association between serum ADA levels and GD among a cohort medium-sized of Chinese participants. The main findings of this study are as follows: first, compared

Table 3 Relationship between ADA and clinical parameters.

\begin{tabular}{|c|c|c|c|c|}
\hline \multirow[b]{2}{*}{ Variables } & \multicolumn{2}{|c|}{ GD } & \multicolumn{2}{|c|}{ HC } \\
\hline & $r$ & $P$ value & $r$ & $P$ value \\
\hline Age & 0.298 & 0.001 & 0.273 & 0.016 \\
\hline Male & 0.043 & 0.653 & 0.118 & 0.305 \\
\hline Smoking & -0.065 & 0.496 & -0.150 & 0.192 \\
\hline Relapsed case & - & - & 0.012 & 0.905 \\
\hline Antithyroid treatment & - & - & -0.043 & 0.656 \\
\hline $\mathrm{BMI}$ & 0.000 & 0.999 & 0.044 & 0.706 \\
\hline SBP & 0.117 & 0.218 & 0.117 & 0.313 \\
\hline DBP & -0.248 & 0.009 & 0.052 & 0.652 \\
\hline TSH & -0.262 & 0.005 & -0.031 & 0.789 \\
\hline FT3 & 0.378 & $<0.001$ & -0.008 & 0.942 \\
\hline FT4 & 0.379 & $<0.001$ & 0.121 & 0.296 \\
\hline TPOAb & 0.209 & 0.029 & -0.087 & 0.510 \\
\hline $\operatorname{TgAb}$ & 0.095 & 0.322 & -0.055 & 0.689 \\
\hline TRAb & 0.518 & $<0.001$ & -0.079 & 0.567 \\
\hline Thyroid VolT & 0.421 & $<0.001$ & 0.386 & 0.052 \\
\hline
\end{tabular}

$r$, Spearman's correlation coefficient. to $\mathrm{HC}$, patients with GD had relatively higher serum ADA levels; second, along with the ADA tertile ascending, serum FT3 levels, FT4 levels, TPOAb levels, TRAb levels, and thyroid VolT gradually increased in GD patients; third, in GD patients, serum ADA levels were positively associated with serum FT3 levels, FT4 levels, TPOAb levels, TRAb levels, and thyroid VolT, and negatively associated with serum TSH levels; while there were no similar correlations observed in $\mathrm{HC}$; fourth, ADA was independently positively associated with ln (TRAb) in GD patients; fifth, the optimal cut-off value of ADA to indicate GD was $10 \mathrm{U} / \mathrm{L}$, and its corresponding sensitivity and specificity were 67.0 and $80.5 \%$, respectively.

Circulation TRAb is the hallmark of GD. By binding to the TSH receptor, TRAb enhances the production of cAMP in thyrocytes, thereby promoting the release of TSH and the growth of thyrocytes, and eventually causing hyperthyroidism and goiter (15). Therefore, the production of TRAb plays the key role in the onset and progression of GD. Dendritic cells (DCs) present antigens to $\mathrm{CD}^{+} \mathrm{T}$ cells which then stimulate the

Table 4 Multiple linear regression analysis with In (TRAb) as the dependent variable in GD subgroup.

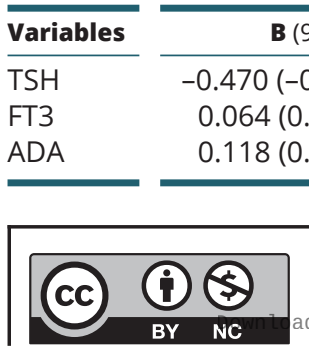

This work is licensed under a Creative Commons Attribution-NonCommercial 4.0 International License. ded from Bioscientifica.com at 04/26/2023 07:32:02AM via free access 


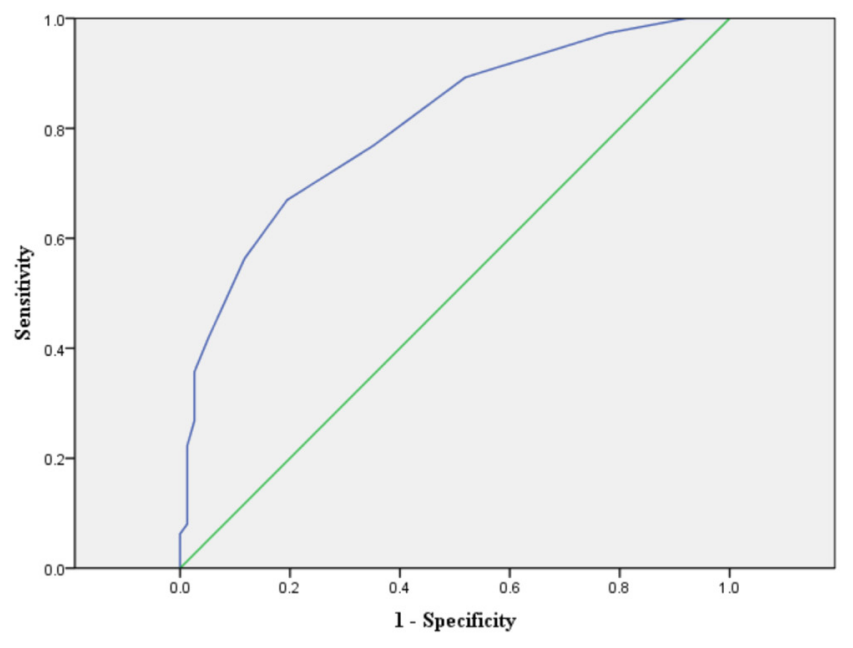

Figure 1

ROC analysis to analyze the ability of ADA to indicate GD.

persistent activation and expansion of $\mathrm{B}$ cells to produce TRAb (16). When subjected to invading pathogen and foreign antigen, immature DCs (iDCs) which are widely distributed among peripheral tissues can be activated. Casanova et al. cultured human iDCs in vitro, and added ADA to the culture medium to enhance the maturation of DCs and render DCs that are more immunogenic (17). For $\mathrm{CD}^{+}{ }^{+} \mathrm{T}$ cells, ADA can interact with $\mathrm{CD} 26$ on the surface of $\mathrm{T}$ cells to trigger co-stimulatory signals of human T cells, suggesting that $\mathrm{ADA}$ is an important modulator of $\mathrm{CD} 4^{+}$ $\mathrm{T}$ cell differentiation (18). Hence, ADA may participate in the onset and progress of GD by stimulating DCs and $\mathrm{T}$ cells to promote the production of TRAb. Consistent with this speculation, the present study found that ADA was an independent contributor to ln (TRAb) in GD patients. In addition, ADA can significantly increase the production of T helper- 1 cells and proinflammatory cells, such as tumor necrosis factor- $\alpha$ (TNF- $\alpha$ ) and interleukin- 6 (IL-6), which may contribute to the exacerbation of GD (19).

In this study, we found that serum ADA levels in GD patients were significantly higher than those in $\mathrm{HC}$, and that serum ADA levels in GD patients were closely related to thyroid function. Untreated hyperthyroidism in GD patients can cause a sort of complications, including weight loss, osteoporosis, thrombosis, atrial fibrillation, brittle fracture, cardiac hypertrophy, and congestive heart failure and so on $(20,21,22)$. In the hypermetabolic state of hyperthyroidism, the increase of intracellular ATP and oxygen consumption and the dysfunction of mitochondrial respiratory chain generate the imbalance of redox balance, resulting in the over-production of reactive oxygen species (ROS) in peripheral tissues (23). A prospective study that enrolled 21 GD patients revealed that oxidative stress indexes were associated with thyroid function, and restoration of euthyroidism could significantly decreased oxidative stress indexes (24). Thus, excessive oxidative stress may be the pathophysiology basis for the occurrence of complications related to GD. Baldissarelli et al. established a hyperthyroid rat model by levothyroxine administration and found that ADA activity in the platelets of the hyperthyroid rat was significantly higher than controls, and ADA levels were positively associated with ROS levels (25). Multiple studies have confirmed the correlation between serum ADA levels and ROS in a variety of diseases (26), and some studies have also confirmed that inhibition of ADA can significantly reduce the production of ROS in vivo (27). The mechanism behind this connection is that ADA can accelerate the differentiation and maturation of T lymphocytes (6). Activated T lymphocytes can produce inflammatory cytokines, thus stimulating macrophage and neutrophil activations, eventually leading to excess production of ROS (28). Thus, ADA may be the bridge linking hyperthyroidism, oxidative stress, and GD complications.

Growing evidences have shown that inhibition of ADA is beneficial for ameliorating some diseases. Pentostatin is a potent ADA inhibitor that, when applied to atherosclerotic mice, exerted anti-atherosclerotic effects by reducing macrophage accumulation and improving endothelial function (29). In hyperthyroidism pig, the administration of pentostatin significantly increased adenosine levels, enhanced A1 adenosinergic system, which in turn exerted negative inotropic effects and might ultimately improve myocardial ischemia (30). Moreover, a review published in 2020 pointed out that multiple commonly used drugs such as simvastatin, metoprolol, and aspirin could significantly inhibit ADA activity at conventional doses (31). Therefore, inhibition of ADA in patients with GD is promising and simple to implement.

However, several limitations of our study were unavoidable. First, as a cross-sectional study, a causeeffect relationship between serum ADA levels and GD cannot be concluded. Second, the generalizability of our study is limited due to that all the subjects enrolled in this study were Chinese. Third, the immune function and oxidative stress indexes of GD patients were not measured simultaneously in this study. Therefore, further research should be conducted to validate the results of our study and to address the above limitations. 


\section{Conclusions}

In summary, this is the first study to demonstrate that serum ADA levels are closely associated with GD. Elevated ADA may be involved in the pathogenesis of GD and the development of complications in GD patients. Future studies need to focus on evaluating the potential of ADA as a target for GD surveillance and treatment.

\section{Declaration of interest}

The authors declare that there is no conflict of interest that could be perceived as prejudicing the impartiality of the research reported.

\section{Funding}

The study was supported by the Medical Research Project of Health Commission of Nantong (MB2020012), the Science and Technology Support Program of Nantong (JC2019068), and the Science and Technology Support Program of Nantong (MS22019007).

\section{Availability of data and materials}

The current data are available to all interested researchers upon reasonable request.

\section{Ethics approval and consent to participate}

The study was approved by the institutional review board of Affiliated Hospital 2 of Nantong University and First People's Hospital of Nantong City, and written informed consent was obtained from all participants.

\section{Author contribution statement}

$C L, W L$, and $F X$ participated in the design of the study, data collection, analysis of the data, and drafting of the manuscript. J $S$ and $C L$ conceived of the study, participated in its design and revised the manuscript. $C L, X G$, and $X W$ participated in the analysis of the data and revised the manuscript. $C L$ and $Y W$ participated in data collection. All authors read and approved the final manuscript.

\section{References}

1 Nyström HF, Jansson S \& Berg G. Incidence rate and clinical features of hyperthyroidism in a long-term iodine sufficient area of Sweden (Gothenburg) 2003-2005. Clinical Endocrinology 201378 768-776. (https://doi.org/10.1111/cen.12060)

2 Brix TH, Kyvik KO \& Hegedüs L. What is the evidence of genetic factors in the etiology of Graves' disease? A brief review. Thyroid $1998 \mathbf{8}$ 727-734. (https://doi.org/10.1089/thy.1998.8.727)

3 Subekti I \& Pramono LA. Current diagnosis and management of Graves' disease. Acta Medica Indonesiana 201850 177-182.

4 Yuan J, Lu X \& Yue Y. Comparison of curative effect of 131I and antithyroid drugs in Graves' disease: a meta-analysis. Minerva Endocrinologica 201843 511-516. (https://doi.org/10.23736/S03911977.17.02551-2)

5 Niraula A, Thapa S, Kunwar S, Lamsal M, Baral N \& Maskey R. Adenosine deaminase activity in type 2 diabetes mellitus: does it have any role? BMC Endocrine Disorders 201818 58. (https://doi.org/10.1186/ s12902-018-0284-9)

6 Kaya S, Cetin ES, Aridogan BC, Arikan S \& Demirci M. Adenosine deaminase activity in serum of patients with hepatitis: a useful tool in monitoring clinical status. Journal of Microbiology, Immunology, and Infection $2007 \mathbf{4 0} 288-292$.

7 Moustafa YM, Elsaied MA, Abd-Elaaty EM \& Elsayed RA. Evaluation of serum adenosine deaminase and inflammatory markers in psoriatic patients. Indian Journal of Dermatology 201964 207-212. (https://doi. org/10.4103/ijd.IJD_420_17).

8 Torgutalp M, Efe C, Babaoglu H \& Kav T. Relationship between serum adenosine deaminase levels and liver histology in autoimmune hepatitis. World Journal of Gastroenterology 201723 3876-3882. (https://doi.org/10.3748/wjg.v23.i21.3876)

9 Yordanova M, Gerova D, Atanassova A \& Galunska B. Adenosine deaminase as a useful biomarker for diagnosis and monitoring of inflammatory bowel disease. Clinical Laboratory 202066 7. (https:// doi.org/10.7754/Clin.Lab.2019.191124)

10 Zamani B, Jamali R \& Jamali A. Serum adenosine deaminase may predict disease activity in rheumatoid arthritis. Rheumatology International 201232 1967-1975. (https://doi.org/10.1007/s00296-0111912-0)

11 Karbownik M, Brzeziańska E, Zasada K \& Lewiński A. Expression of genes for certain enzymes of pyrimidine and purine salvage pathway in peripheral blood leukocytes collected from patients with Graves' or Hashimoto's disease. Journal of Cellular Biochemistry 200389 550-555. (https://doi.org/10.1002/jcb.10533)

12 Karbownik M, Zasada K, Wyczechowska D, Lewiński A \& FabianowskaMajewska K. Purine metabolism in leukocytes and erythrocytes in Graves' or Hashimoto's disease. Endocrine Research 200228 207-215. (https://doi.org/10.1081/erc-120015059)

13 Köse K, Utaş S, Yazici C, Akdaş A \& Keleștimur F. Effect of propylthiouracil on adenosine deaminase activity and thyroid function in patients with psoriasis. British Journal of Dermatology 2001 144 1121-1126. (https://doi.org/10.1046/j.1365-2133.2001.04221.x)

14 Falkowski B, Szczepanek-Parulska E, Krygier A, Wrotkowska E \& Ruchala M. Evaluation of interleukin-29 in autoimmune and inflammatory thyroid diseases. Clinical Endocrinology 202194 998-1003. (https://doi.org/10.1111/cen.14418)

15 Rapoport B \& McLachlan SM. TSH receptor cleavage into subunits and shedding of the a-subunit; a molecular and clinical perspective. Endocrine Reviews 201637 114-134. (https://doi.org/10.1210/er.20151098)

16 Wang Q, Shi BM, Xie F, Fu ZY, Chen YJ, An JN, Ma Y, Liu CP, Zhang XK \& Zhang XG. Enhancement of CD4(+) T cell response and survival via coexpressed OX40/OX40L in Graves' disease. Molecular and Cellular Endocrinology 2016430 115-124. (https://doi.org/10.1016/j. mce.2016.04.008)

17 Casanova V, Naval-Macabuhay I, Massanella M, Rodríguez-García M, Blanco J, Gatell JM, García F, Gallart T, Lluis C, Mallol J, et al. Adenosine deaminase enhances the immunogenicity of human dendritic cells from healthy and HIV-infected individuals. PLOS ONE 20127 e51287. (https://doi.org/10.1371/journal.pone.0051287)

18 Martinez-Navio JM, Casanova V, Pacheco R, Naval-Macabuhay I, Climent N, Garcia F, Gatell JM, Mallol J, Gallart T, Lluis C, et al. Adenosine deaminase potentiates the generation of effector, memory, and regulatory CD4+ T cells. Journal of Leukocyte Biology 201189 127-136. (https://doi.org/10.1189/jlb.1009696)

19 Pacheco R, Martinez-Navio JM, Lejeune M, Climent N, Oliva H, Gatell JM, Gallart T, Mallol J, Lluis C \& Franco R. CD26, adenosine deaminase, and adenosine receptors mediate costimulatory signals in the immunological synapse. PNAS 2005 102 9583-9588. (https://doi. org/10.1073/pnas.0501050102)

20 Kahaly GJ, Bartalena L, Hegedüs L, Leenhardt L, Poppe K \& Pearce SH. 2018 European Thyroid Association guideline for the management https://ec.bioscientifica.com https://doi.org/10.1530/EC-21-0344 (c) 2021 The authors Published by Bioscientifica Ltd

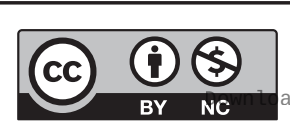

This work is licensed under a Creative Commons Attribution-NonCommercial 4.0 International License. ded from Bioscientifica.com at 04/26/2023 07:32:02AM 
of Graves' hyperthyroidism. European Thyroid Journal 20187 167-186. (https://doi.org/10.1159/000490384)

21 Kahaly GJ \& Dillmann WH. Thyroid hormone action in the heart. Endocrine Reviews 200526 704-728. (https://doi.org/10.1210/er.20030033)

22 Biondi B \& Kahaly GJ. Cardiovascular involvement in patients with different causes of hyperthyroidism. Nature Reviews. Endocrinology 2010 6 431-443. (https://doi.org/10.1038/nrendo.2010.105)

23 Halliwell B. Reactive oxygen species in living systems: source, biochemistry, and role in human disease. American Journal of Medicine 199191 14S-22S. (https://doi.org/10.1016/0002-9343(91)90279-7)

24 Larsen CB, Riis KR, Winther KH, Larsen EL, Ellervik C, Hegedüs L, Brix TH, Poulsen HE \& Bonnema SJ. Treatment of hyperthyroidism reduces systemic oxidative stress, as measured by markers of RNA and DNA damage. Journal of Clinical Endocrinology and Metabolism 2021 106 e2512-e2520. (https://doi.org/10.1210/clinem/dgab273)

25 Baldissarelli J, Santi A, Schmatz R, Martins CC, Zanini D, Reichert KP, Thomé GR, Palma TV, da Costa P, Morsch VM, et al. Hypothyroidism and hyperthyroidism change ectoenzyme activity in rat platelets. Journal of Cellular Biochemistry 2018119 6249-6257. (https://doi. org/10.1002/jcb.26856)

26 Köse K, Yazici C \& Aşşioğlu O. The evaluation of lipid peroxidation and adenosine deaminase activity in patients with Behçet's disease. Clinical Biochemistry 200134 125-129. (https://doi.org/10.1016/s00099120(01)00190-4)
27 Uzar E, Sahin O, Koyuncuoglu HR, Uz E, Bas O, Kilbas S, Yilmaz HR, Yurekli VA, Kucuker H \& Songur A. The activity of adenosine deaminase and the level of nitric oxide in spinal cord of methotrexate administered rats: protective effect of caffeic acid phenethyl ester. Toxicology 2006218 125-133. (https://doi.org/10.1016/j. tox.2005.10.014)

28 Erkiliç K, Evereklioglu C, Cekmen M, Ozkiris A, Duygulu F \& Dogan H. Adenosine deaminase enzyme activity is increased and negatively correlates with catalase, superoxide dismutase and glutathione peroxidase in patients with Behçet's disease: original contributions/ clinical and laboratory investigations. Mediators of Inflammation 2003 12 107-116. (https://doi.org/10.1080/0962935031000097754)

29 Kutryb-Zajac B, Mierzejewska P, Slominska EM \& Smolenski RT. Therapeutic perspectives of adenosine deaminase inhibition in cardiovascular diseases. Molecules 202025 4652. (https://doi. org/10.3390/molecules25204652)

30 Pak K, Zsuga J, Kepes Z, Erdei T, Varga B, Juhasz B, Szentmiklosi AJ \& Gesztelyi R. The effect of adenosine deaminase inhibition on the A1 adenosinergic and M2 muscarinergic control of contractility in euand hyperthyroid guinea pig atria. Naunyn-Schmiedeberg's Archives of Pharmacology 2015388 853-868. (https://doi.org/10.1007/s00210-0151121-6)

31 Bagheri S, Saboury AA \& Haertlé T. Adenosine deaminase inhibition. International Journal of Biological Macromolecules 2019141 1246-1257. (https://doi.org/10.1016/j.ijbiomac.2019.09.078)

Received in final form 16 August 2021

Accepted 2 September 2021

Accepted Manuscript published online 2 September 2021
This work is licensed under a Creative Commons Attribution-NonCommercial 4.0 International License. ded from Bioscientifica.com at 04/26/2023 07:32:02AM 